\title{
The Effect of Process Parameters on Surface Behaviors of Low Carbon Steels Modified by Pulse Plasma Technique
}

\author{
G. AgDAŞ AND Y.Y. ÖZBEK* \\ Sakarya University, Engineering Faculty, Department of Metallurgical and Materials Engineering \\ 54187, Esentepe Campus, Sakarya, Turkey
}

\begin{abstract}
In this study, microstructure and surface properties of low carbon steels (St52) treated by pulse plasma were investigated. The surfaces of samples were modified by pulse plasma technique. Four different plasma gun nozzle distances as $50 \mathrm{~mm}, 60 \mathrm{~mm}, 70 \mathrm{~mm}$, and $80 \mathrm{~mm}$ and one battery capacity were chosen for surface modification. Cross-section microstructures of samples were investigated and modified layer thicknesses were measured by optical microscope. The thickness values of modified layer were obtained in a range from $10 \mu \mathrm{m}$ to $41 \mu \mathrm{m}$. The samples were immersed into liquid nitrogen and then breaked in a Charpy machine. The fractured surfaces were exposed to scanning electron microscopy analyses. At the end of the study, thin grains and small tungsten grains coming from tungsten electrode were detected. After pulse plasma treatment, new structures and new phases and high hardness values were obtained.
\end{abstract}

DOI: 10.12693/APhysPolA.125.393

PACS: 81.65.-b

\section{Introduction}

The interaction of intense-pulsed energetic beams such as ion, electron, and laser beams with materials and its application in industry have attracted much attention over the past few decades [1-3]. Indeed, each pulse of concentrated energy flux causes super-fast thermal cycles and modifies the surface in a way to impart the material with improved physicochemical and strength properties that are often unattainable with other more conventional surface treatment methods [1-4]. These non-equilibrium processes can easily change the microstructure, chemical composition, phase structure, and stress state at the surface.

The pulsed-plasma technology allows a simultaneous, in one treatment pulse, realization of different methods of affecting the workpiece surface: elasto-plastic deformation, impact by sound and pulsed magnetic field, heat and electric-pulse treatment, and deformation of metals and alloys during reversible $(\alpha+\gamma)$ transformations. High power density of the flow (up to $10^{7} \mathrm{~W} / \mathrm{cm}^{2}$ at the point of contact with the workpiece surface) makes it possible to perform treatment in air atmosphere with no surface preparation. Treatment with a high-energy density flow of alloying elements causes no changes in geometric sizes of workpieces. Therefore, it is suggested that it should be used as a finishing operation. Industrial verification shows that performance of tools and machine parts after modification of their working surfaces increases $3-5$ times. Depending upon the composition of the high-energy density flow, the surface layer can have high anti-friction properties, as well as high heat, wear, and corro-

*corresponding author; e-mail: yyarali@sakarya.edu.tr sion resistance. Along with reduction in cost of a workpiece material, this leads to reduction in machining and heat treatment costs $[3-5]$.

The main aim of this work was studying of surface properties of St52 steel modified by pulse plasma treatment. The surface properties are dependent parameters of pulse treatment. In this study, different plasma parameters were applied to the steel surfaces to investigate the effect of number of pulses and nozzle distance. After treatment, the cross-sectional microstructures of specimens were observed.

\section{Experimental procedure}

A standard low carbon, St52 steel has been used in the study and the chemical composition of the steel is given in Table $\mathrm{I}$.

The specimens were cut out from square bars and subsequently machined and grinded in order to obtain the desired shape and surface roughness. Before pulse plasma treatments all samples were polished progressively to 1200 grit with grinding paper to obtain identical surface conditions. The pulse plasma treatment was done only on work surface of samples. The pulse plasma parameters were shown in Table II.

The chemical composition of St 52 steel.

TABLE I

\begin{tabular}{c|c|c|c|c|c|c|c|c}
\hline \hline [wt\%] & $\mathrm{C}$ & $\mathrm{Si}$ & $\mathrm{Mn}$ & $\mathrm{P}<$ & $\mathrm{S}<$ & $\mathrm{Cr}$ & $\mathrm{Mo}$ & $\mathrm{Ni}$ \\
\hline St 52 & 0.22 & 0.55 & 1.60 & 0.04 & 0.035 & 0.06 & 0.02 & 0.1
\end{tabular}

The morphology and cross-sectioned microstructure of samples were studied by optic microscope. The phase compositions of the modified surface were investigated by X-ray diffractometry (XRD) using a Rigaku6600. Micro-hardness test machine was used to measure the sur- 
TABLE II

The parameters of pulse plasma treatment.

\begin{tabular}{c|c|c|c|c}
\hline \hline $\begin{array}{c}\text { Sample } \\
\text { No. }\end{array}$ & $\begin{array}{c}\text { Distances } \\
{[\mathrm{mm}]}\end{array}$ & Electrode & $\begin{array}{c}\text { Number } \\
\text { of pulse }\end{array}$ & $\begin{array}{c}\text { Battery } \\
\text { capacity } \\
{[\mathrm{mF}]}\end{array}$ \\
\hline 1 & 50 & Mo & 5 & 800 \\
2 & 50 & Mo & 10 & 800 \\
3 & 50 & Mo & 15 & 800 \\
4 & 60 & Mo & 5 & 800 \\
5 & 60 & Mo & 10 & 800 \\
6 & 60 & Mo & 15 & 800 \\
7 & 70 & Mo & 5 & 800 \\
8 & 70 & Mo & 10 & 800 \\
9 & 70 & Mo & 15 & 800 \\
10 & 80 & Mo & 5 & 800 \\
11 & 80 & Mo & 10 & 800 \\
12 & 80 & Mo & 15 & 800
\end{tabular}

face and subsurface microhardness at a load of $50 \mathrm{~g}$ (HV0.050).

After pulse plasma process, notched specimens were immersed into liquid nitrogen for one minute and then they were cracked by hammer from notch region. After that, the fractured regions were analysed by SEM.

\section{Results and discussion}

Figure 1 shows the optical micrographs of the sample for number of pulse 5, 10, 15 in $60 \mathrm{~mm}$ distance between the nozzle and sample. The metastable structures are formed on the modified surface under non-equilibrium condition. The modified zone is shown clearly.

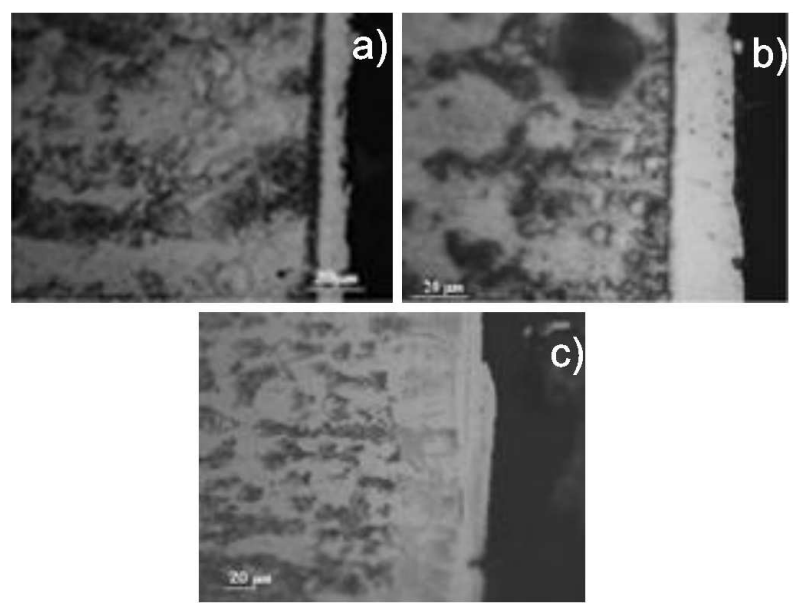

Fig. 1. Micrograph of samples treated by pulse plasma (a) 5 pulse, (b) 10 pulse, (c) 15 pulse (in $60 \mathrm{~mm}$ distances).

The thicknesses of modified layers are indicated in Fig. 2. The thicknesses of modified layers are changed with pulse plasma parameters.

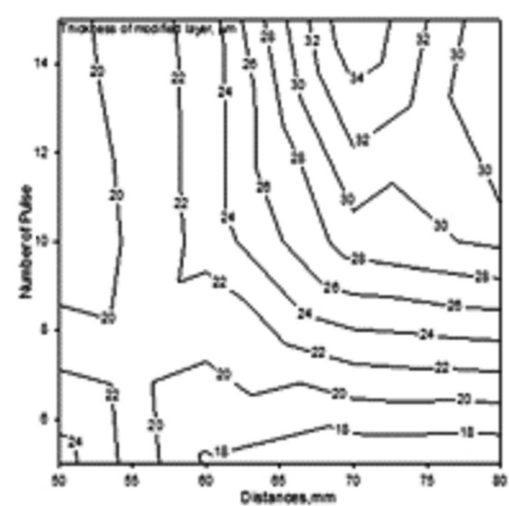

Fig. 2. The relationship thickness of between modified layer and pulse plasma parameters.

There exists a thin layer on the top surface, which shows a completely different contrast from the rest of the material. This layer corresponds to the melted layer. This layer can be divided into different zones having different contrasts. The thickness of the melted layers is about $10-40 \mathrm{~mm}$. The difference in contrast between the bulk material and the melted layer is due to the fact of the fast solidification in the melted layer. However, these leads to the formation of more homogeneous and ultrafine microstructure. The upper part corresponds to the melted layer and its depth increases when the number of pulses increases from 5 to 15 .

The XRD traces recorded from the surfaces before and after the pulse plasma treatment are shown in Fig. 3. From the surface microstructure and the XRD results, it can be deduced that new phases were formed on surface. Also interesting in Fig. 3 is the significant change in relative intensity of the diffraction peaks between the initial and the pulse plasma treated samples. Their peak intensity increases with the number of pulses, indicating that the volume fraction of the $\gamma$ phase is detected by XRD increases [4].

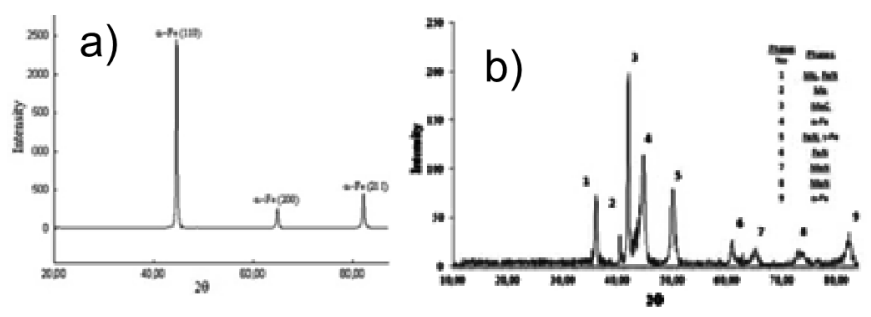

Fig. 3. The XRD results of (a) untreatment samples, (b) after pulse plasma treatment (samples 5).

Indeed, the new grains of phase that nucleated from the melt must grow along the thermal gradient direction (i.e. perpendicularly to the treated surface) following specific crystallographic directions. Finally, even without solidification texture or phase transformation, it has been established that the thermal stresses generated during the 
fast cooling induce the activation of deformation mechanisms that can produce texture changes on the surface of the pulse plasma treated samples [5].

The untreatment sample hardness values were $170 \mathrm{HV}$, but pulse plasma treatment was increased five times from untreatment sample (Fig. 4). The amount of these strong phases precipitated in the matrix phase was one of the reasons in leading to increase in hardness values of the treated samples. Phase and structure transformations result in the modification of surface characteristics. The increase of the pulse number or that of the energy density absorbed by the surface lead to the increase in the microhardness values.

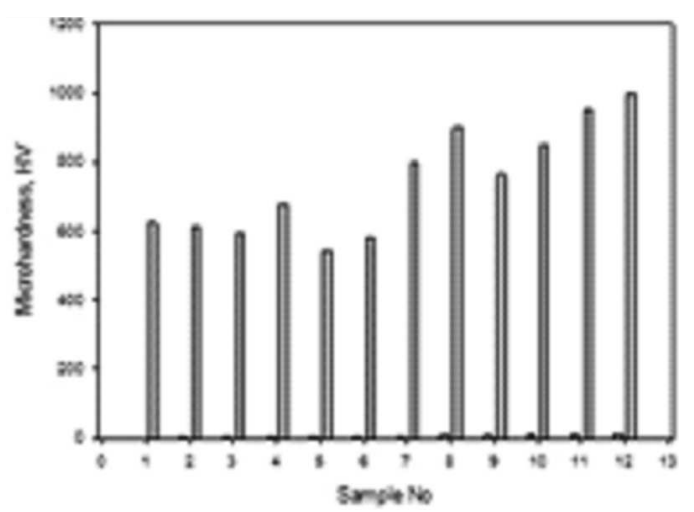

Fig. 4. The microhardness values of modified zone after pulse plasma.

Another reason for the increase in hardness values of the samples was the decreased grain size due to the increased cooling rate within the cycles of pulses and in the present study, for the samples subjected to pulse plasma treatment with the higher the number of pulses, the lower the grain size and the higher the microhardness values [6].
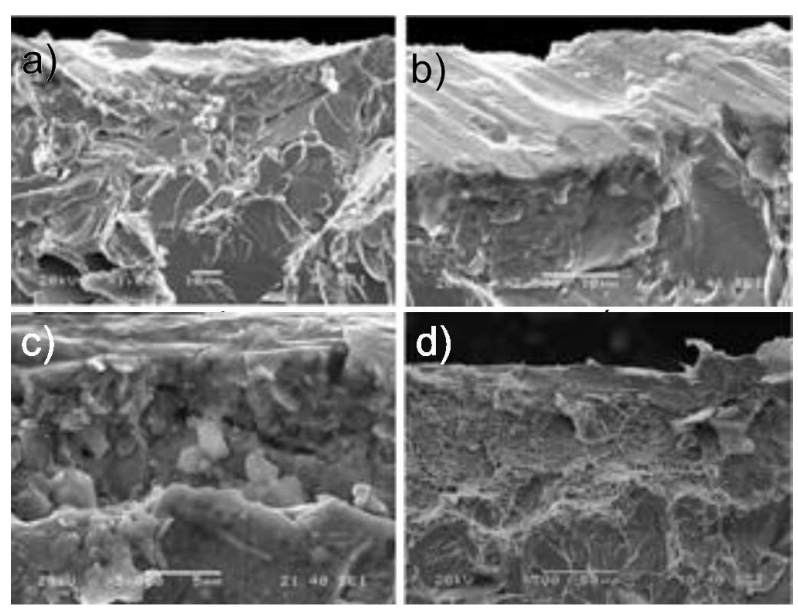

Fig. 5. The fracture surfaces of (a) sample 2, (b) sample 3, (c) sample 4, (d) sample 5.
It is clearly visible that for both specimens the fracture mechanism at the initiation site is identical, namely transgranular (quasi-) cleavage. The cleavage fracture mode is mainly observed throughout the whole specimen, but some ductile dimpled fracture mode is also found in the cold-drawn bar, as indicated by dotted oval areas in Fig. 5a and b. The zone of localized plastic deformation in the vicinity of the fatigue crack tip was followed by a region of stable crack propagation characterized by dimple morphology. This supports the increased impact energy in the cold-drawn bar and indicates the increased resistance to crack propagation in lamellar-structured materials [4]. Also the oxide grains were observed on the surface (Fig. 5c). The laminated structure composed in surface. The ductile fracture was formed in modified layer (Fig. 5d). The transgranular fracture was shown in substrate.

\section{Conclusion}

In summary, it is clear that the pulse plasma treatment induces drastic temperature gradients in the surface of the material and that, concomitantly to the thermal effect, the pulse electron beam creates a dynamic stress field that causes intense deformation at the material surface. The number of pulses of these treatments is also shown to affect the microstructure. The formation of a homogeneous layer is obtained when increasing the number of pulses. The new phases were formed on modified surface. Pulse plasma process resulted in high surface hardness values (five times increasing) in short processing times with a rather simple operation. This process is very simple, easy, and cheap for improving surface properties.

\section{References}

[1] A.D. Pogrebnjak, V.T. Shablya, N.V. Sviridenko, A.N. Valyaev, S.V. Plotnikov, M.K. Kylyshkanov, Surf. Coat. Technol. 111, 46 (1999).

[2] Y. Hao, B. Gao, G.F. Tu, H. Cao, S.Z. Hao, C. Dong, Appl. Surf. Sci. 258, 2052 (2012).

[3] A.D. Pogrebnjak, V.S. Ladysev, N.A. Pogrebnjak, A.D. Michaliov, V.T. Shablya, A.N. Valyaev, Vacuum 58, 45 (2000)

[4] K.M. Zhang, J.X. Zou, B. Bolle, T. Grosdidier, Vacuum 87, 60 (2013).

[5] K. Hyunmin, K. Minju, J. Hyeok, S. Hyoung, K. Seop, M.B. Chul, L. Sunghak, Mater. Sci. Eng. A 571, 38 (2013).

[6] J.X. Zou, T. Grosdidier, K.M Zhang, C. Dong, Appl. Surf. Sci. 255, 4758 (2009). 